\title{
COMPLEX COBORDISM AND EMBEDDABILITY OF CR-MANIFOLDS
}

\author{
Bruno De Oliveira
}

This paper studies (directed) complex cobordisms between compact three dimensional strictly pseudoconvex Cauchy-Riemann manifolds. Assume the complex cobordism between the compact strictly pseudoconvex Cauchy-Riemann manifolds $M_{1}$ and $M_{0}$ is given by a compact complex manifold $X$ with boundary having $M_{1}$ as its pseudoconvex end and $M_{0}$ as its pseudoconcave end. We answer the following questions: if one of the ends is an embeddable CR-manifold is the other end also embeddable? Are all CR-functions on the pseudoconvex end of $X$ boundary values of holomorphic functions on the interior of $X$ ? If the complex cobordism manifold $X$ is of dimension greater than 2 , the affirmative answer to these questions follows immediately from the embeddability of all strictly pseudoconvex CR-manifolds of dimension greater than 3. For complex cobordisms of dimension 2 these questions were still open. The ends of complex cobordism 2-manifolds are strictly pseudoconvex CR-manifolds of dimension 3 whose embeddability is no longer guaranteed and is a topic of active research.

To answer the questions raised above, we produce two new methods to construct pseudoconcave surfaces, whose boundaries are non-embeddable strictly pseudoconvex CR-manifolds of dimension 3. The constructions allow us to show that:

1. There are complex cobordant 3-dimensional strictly pseudoconvex CRmanifolds $M_{1}$ and $M_{0}$, with one embeddable and the other not.

2. There are complex cobordism 2-manifolds $X$ with CR-functions on the pseudoconvex end $M_{1}$ that are not boundary values of holomorphic functions on the interior of $X$.

3. There are complex cobordism 2-manifolds $X$ with embeddable pseudoconvex ends $M_{1}$, where the extendabitity of all the CR-functions on $M_{1}$ to holomorphic functions on $X$ does not imply the embeddability of the pseudoconcave end $M_{0}$.

4. There are holomorphic families of complex cobordism 2-manifolds where all the members have their pseudoconvex ends embeddable but only the central member has the pseudoconcave end embeddable.

The first result answers negatively the main question of this paper on the preservation of embeddability under complex cobordisms. In the literature this

Received August 17, 2001, Revised May 22, 2003.

Partially supported by NSF Postdoctoral Research Fellowship DMS-9902393. 
question appeared in [EpHe1-00]. Previous to our result, the only answer was positive [EpHe01], but the complex cobordism manifold $X$ had an extra condition. It was required that $X$ had a strictly plurisubharmonic function $\varphi$ and that its ends $M_{1}$ and $M_{0}$ were level sets of $\varphi$.

The second result gives the first case where CR-functions on the pseudoconvex end $M_{1}$ of a complex manifold $X$, whose ends are strictly pseudoconvex CR-manifolds, do not extend to holomorphic functions on $X$. The basic results on extendability are in the other direction: the local extendability result of Lewy states that all CR-functions of the pseudoconvex end $M_{1}$ extend to an holomorphic function on a small neighborhood of $M_{1}$ and the global extendability result of Kohn and Rossi [KoRo65] implying that if all the other ends of $X$ are embeddable then all the CR-functions on $M_{1}$ extend. This last global result shows in particular that result 2) is only possible in dimension 2 .

The third result clarifies the strength of the connection between the embeddability of the pseudoconcave end $M_{0}$ of a complex cobordism manifold $X$ having an embeddable pseudoconvex end $M_{1}$ and the extendability of all the CR-functions of $M_{1}$ to holomorphic functions on $X$. The extendability of the CR-functions from the pseudoconvex end $M_{1}$ is necessary for embeddability of $M_{0}$, as follows from [KoRo65], but the result states that it is not sufficient. The fourth result states that the property that a complex cobordism manifold preserves embeddability is not stable under small deformations. The last two results also say that complex cobordism manifolds not preserving embeddability can be quite similar to complex cobordism manifolds that do.

A compact $(2 \mathrm{n}+1)$-dimensional Cauchy - Riemann manifold (CR- $(2 \mathrm{n}+1)$ manifold) consists of: a compact $(2 \mathrm{n}+1)$-dimensional manifold $M$, a rank $\mathrm{n}$ complex subbundle $T^{0,1} M \subset T M \otimes \mathbb{C}$ satisfying $T^{1,0} M \cap T^{0,1} M=\{0\}\left(T^{1,0} M \equiv\right.$ $\left.\overline{T^{0,1} M}\right)$ and the integrability condition $\left.\left[\bar{Z}, \overline{Z^{\prime}}\right]\right|_{p} \in T_{p}^{0,1} M$ for local sections $\bar{Z}, \overline{Z^{\prime}}$ of $T^{0,1} M$. If the CR- $(2 \mathrm{n}+1)$-manifold has the additional property that any nonvanishing local section $\bar{Z}$ of $T^{0,1} M$ is such that $\left.[Z, \bar{Z}]\right|_{p} \notin T_{p}^{0,1} M \oplus T_{p}^{1,0} M$ for any $p \in M$, it is called strictly pseudoconvex (SPCR- $(2 \mathrm{n}+1)$-manifold).

A differentiable function $f: M \rightarrow \mathbb{C}$ is said to be a $C R$ - function if it is in the kernel of the $\bar{\partial}_{b}$ operator, $\bar{\partial}_{b} f=\left.d f\right|_{T^{0,1} M}$. That is, it verifies the tangential Cauchy-Riemann equations $\bar{Z} f=0$ for all local sections of $T^{0,1} M$.

Definition. A CR- $(2 \mathrm{n}+1)$-manifold $M$ is embeddable if there is a collection of CR functions $\left\{f_{1}, \ldots, f_{N}\right\}$ giving an embedding $f=\left(f_{1}, \ldots, f_{N}\right): M \rightarrow \mathbb{C}^{N}$.

The origin of CR-structures lies in the study of real hypersurfaces in $\mathbb{C}^{n}$. The CR-structure being given by $T^{0,1} M=T^{0,1} \mathbb{C}^{n} \cap T M \otimes \mathbb{C}$. A CR-function is a function that satisfies the differential conditions of holomorphy that can be verified along M. An important example of SPCR-manifolds are the boundaries of strictly pseudoconvex domains in $\mathbb{C}^{n}$.

Definition. A CR- $(2 \mathrm{n}+1)$-manifold $M$ is fillable if it is the boundary of a normal complex Stein space $X$ with the CR-structure induced from the complex 
structure of $X$. A filling of $M$ is any normal complex space $Y$ which is a modification of a Stein space and whose boundary is $M$.

An example of Andreotti and Grauert, see [Ro65] showed that not all compact SPCR-3-manifolds are embeddable. Later Boutet de Monvel [Bou74] showed that for $n \geq 2$ any compact SPCR- $(2 \mathrm{n}+1)$-manifold $M$ is embeddable, this follows from the result of Kohn stating that the range of $\bar{\partial}_{b}$ is closed plus the result of [Bou74] stating that if the range of $\bar{\partial}_{b}$ is closed then $M$ is embeddable. The following important result also holds: fillability of any compact SPCR$(2 \mathrm{n}+1)$-manifold $M$ is equivalent to the embeddability of $M$. This result follows from [Bou74], [Ko86] and also [HaLa75]. A significant amount of work has been produced to understand the structure of the set of embeddable SPCR-structures for 3-manifolds by Bland, Burns, Catlin, Epstein, Henkin and Lempert.

\section{Directed complex cobordisms}

The CR-structure on a smooth real hypersurface $M$ given as a level set of a strictly plurisubharmonic function $\varphi$ on a complex manifold $V, M=\left\{\varphi^{-1}(c)\right\}$ and $d \varphi(p) \neq 0$ on $T_{p} M$ for all $p \in M$, is strictly pseudoconvex. In the opposite direction, let $M$ be a compact smooth boundary component of complex manifold with boundary $X$ such that the induced CR-structure on $M$ is strictly pseudoconvex then $M$ is a nondegenerate level set of a strictly plurisubharmonic function $\varphi$ defined in a neighborhood of $M, V \subset X . M$ is said to be a pseudoconvex (pseudoconcave) end of $X$ if $M=\left\{\varphi^{-1}(0)\right\}$ and $X \cap V=\{\varphi(x) \leq$ $0\}(X \cap V=\{\varphi(x) \geq 0\})$.

Definition. Let $X$ be a connected compact complex manifold with a smooth boundary. If $\partial X=M_{1} \amalg M_{0}$ with $M_{0}$ a disjoint union of pseudoconcave ends and $M_{1}$ a disjoint union of pseudoconvex ends, then $M_{0}$ is said to be complex cobordant to $M_{1}$ and $X$ is a (directed) complex cobordism manifold.

A special case of (directed) complex cobordisms was considered in [EpHe01]. Two CR-manifolds $M_{1}$ and $M_{0}$ are called strictly CR-cobordant, if there is complex cobordism manifold $X$ with $\partial X=M_{1} \amalg M_{0}$ having a strictly plurisubharmonic function, $\phi: X \rightarrow \mathbb{R}$, with $M_{1}$ and $M_{0}$ as level sets $\phi^{-1}(1)$ and $\phi^{-1}(0)$ respectively. $X$ is called a strict complex cobordism manifold. From now on, all complex cobordisms will be directed complex cobordisms.

A compact complex manifold with a smooth boundary is called a pseudoconvex (pseudoconcave) manifold if all its boundary components are pseudoconvex (pseudoconcave) ends. In [KoRo65] it is shown that a pseudoconvex manifold has only one end. This implies that a complex cobordism manifold $X$ of dimension greater than 2 can not have more than one pseudoconvex end. The complex manifold $X^{\prime}$ obtained from $X$ by filling its pseudoconcave ends is pseudoconvex. In Corollary 1, we show that for dimension 2 it is possible to have complex cobordisms manifolds with more than one pseudoconvex ends.

On the other hand it is possible for a pseudoconcave manifold $Y$ to have more than one end. Note that if $Y$ is a pseudoconcave manifold and $M$ and $M^{\prime}$ 
are SPCR-manifolds such that $\partial Y=M \amalg M^{\prime}$, the above definition of directed cobordism does not apply and $M$ is not considered to be complex cobordant to $M^{\prime}$ or vice-versa.

The main goal of this paper is to understand how the embeddability of one end of a complex cobordism manifold $X$ influences the embeddability of the other end. The first observation is that if the pseudoconcave end $M_{0}$ of $X$ is embeddable then the pseudoconvex end $M_{1}$ is also embeddable. The embeddability of $M_{1}$ follows from its fillability, the manifold $S^{\prime}=X \amalg_{M_{0}} S, S$ a filling of $M_{0}$, is a filling of $M_{1}$. The other direction is the interesting case.

Prior to our work, the existing result on whether the embeddability of the pseudoconvex end implies the embeddability of the pseudoconvex end was in the positive direction. The result was for the special case of strictly CR-cobordant SPCR-manifolds [EpHe01]. In [EpHe01] it was shown that if the SPCR-manifolds $M_{1}$ and $M_{0}$ are strictly CR-cobordant then $M_{1}$ is embeddable iff $M_{0}$ is embeddable. One of the main ingredients of the proof is that by using the Lewy extension result all the CR-functions on $M_{1}$ are boundary values of holomorphic functions on $X$. In the next two sections we show that for a general complex cobordism $M_{1}$ being embeddable does not imply that $M_{0}$ is embeddable, and the same holds even if all CR-functions on $M_{1}$ are boundary values of holomorphic functions on the complex cobordism manifold.

It was mentioned in the introduction that the fillability is intimately related to the embeddability for SPCR-manifolds. It is therefore fruitful to relate a complex cobordism manifold with the fillings of its pseudoconvex end.

Definition. Let $M_{1}$ be an embeddable SPCR-manifold. If $M_{1}$ is the pseudoconvex end of a complex cobordism manifold $X$ whose other ends are pseudoconcave, then $X$ is called a partial filling of $M_{1}$. We say that we have rigidity on the partial fillings of $M_{1}$ if they are all modifications of open subsets of the unique Stein normal space filling $M_{1}$.

Let $M_{1}$ be an embeddable SPCR-manifold of dimension greater than 3 then all partial fillings extend to an actual filling of $M_{1}$ and they are rigid. Let $X$ be a complex cobordism manifold with the pseudoconvex end $M_{1}$ and the pseudoconcave ends $M_{1} \amalg \ldots \amalg M_{k}$. The embeddability of all SPCR-manifolds of dimension greater than 3 implies that the $M_{i}, i=1, \ldots, k$, are embeddable or equivalently fillable, then $X$ is a subset of a filling $S^{\prime}$ of $M_{1}, S^{\prime}=X \amalg_{\left(M_{0} \amalg \ldots \amalg M_{k}\right)}$ $\left(S_{1} \amalg \ldots \amalg S_{k}\right)$ with $S_{i}$ a filling of $M_{i}$. In [Gr62] it is shown that all fillings of $M_{1}$ are proper modifications of its unique Stein normal filling.

The question of embeddability being preserved under complex cobordisms can be translated to: can we start filling an embeddable SPRC-3-manifold $M_{1}$ in the wrong way (not extendable to a filling)? A distinct but also relevant question is: what is the freedom in the partial fillings of $M_{1}$ ? Theorem 1 of the next section answers the first question affirmatively and from Theorem 3 of section 3 it follows that there can be plenty of freedom in the partial fillings of $M_{1}$. 
Remark. It is interesting to re-examine the result of Epstein and Henkin [EpHe01] described above. The result implies that, regardless of the dimension, in any partial filling of $M_{1}$ there is a sufficiently small collar of $M_{1}$ in $X$ that is rigid.

\section{Embeddability is not preserved}

In this section we give a method to construct complex cobordisms where one end is embeddable but the other is not. We also show that the complex cobordisms produced by this method are very different, in terms of function theory, from complex cobordisms where all ends are embeddable.

The next lemma will be used to construct pseudoconcave surfaces containing disjoint positive curves.

Lemma 1. Let $X$ be a nonsingular complex surface containing the normal crossing divisor $C=C_{1} \cup \ldots \cup C_{k}$, with $C_{i}$ nonsingular positive compact curves, i.e. $C_{i}^{2}>0$. Then there exist a neighborhood $W$ of $C$ where the line bundle $\mathcal{O}(C)$ associated to the divisor $C$ is positive and a smooth strictly plurisubharmonic function $\varphi: W \backslash C \rightarrow \mathbb{R}$ such that:

1. For every sequence $\left(x_{n}\right)_{n \in \mathbb{N}}, x_{n} \in W \backslash C$ converging to $x \in C$,

$$
\lim _{x_{n} \rightarrow x} \varphi\left(x_{n}\right)=+\infty \text {. }
$$

2. For $c \gg 0, X_{c}=\{x \in W: \varphi(x) \geq c$ or $x \in C\}$ is a relatively compact pseudoconcave neighborhood of $C$.

Proof. First we show that if the line bundle $\mathcal{O}(C)$ is positive in a neighborhhod $W$ of $C$ then we can construct a strictly plurisubharmonic function $\varphi: W \backslash C \rightarrow \mathbb{R}$ satisfying i) and ii). A line bundle $L$ over $X$ is positive at $x \in X$ if $L$ has a hermitean metric $h$ such that the associated real $(1,1)$-form $\Omega_{h}=\sqrt{-1} \Theta_{h}=$ $-\sqrt{-1} \partial \bar{\partial} \log h$ is positive at $x\left(\Theta_{h}\right.$ is the curvature form for $\left.h\right)$.

The line bundle $\mathcal{O}(C)$ is defined by the transition functions $g_{i j}=\frac{f_{j}}{f_{i}}$, where $\left\{f_{i} \in O\left(U_{i}\right)\right\}$ are the defining equations of $C$ for the covering $\left\{U_{i}\right\}$ of $X$. A hermitian metric $h$ on $\mathcal{O}(C)$ is given by a collection of positive functions, $h_{i}$ : $U_{i} \rightarrow \mathbb{R}$ satisfying $h_{j}=h_{i} \frac{\left|f_{i}\right|^{2}}{\left|f_{j}\right|^{2}}$ on $U_{i} \cap U_{j}$. If the hermitian metric $h$ on $O(C)$ is such that the form $\Omega_{h}$ positive at all $x \in W$, then the function $\varphi: W \backslash C \rightarrow \mathbb{R}$ defined locally by:

$$
\left.\varphi\right|_{U_{i} \backslash\left\{f_{i}=0\right\}} \equiv \varphi_{i}=-\log \left(h_{i}\left|f_{i}\right|^{2}\right),
$$

is strictly plurisubharmonic and satisfies the properties i) and ii). The positivity of the form $\Omega_{h}$ on $W$ implies that $\varphi$ is strictly plurisubharmonic on $W \backslash C$. Since $\partial \bar{\partial} \varphi_{i}=\partial \bar{\partial}\left(-\log \left(h_{i}\right)\right)$, where $f_{i} \neq 0$, the positive definiteness of the Levi form of $\varphi_{i}$ follows from the positivity of the $(1,1)$-form $\Omega_{h}$ associated with the metric $h$. The properties i) and ii) follow directly from the definition of $\varphi$ plus the compactness of the $C_{i}$ 's.

We proceed to show that $\mathcal{O}(C)$ is positive in a neighborhood of $C$. The first step is to construct a metric $h$ on $\mathcal{O}(C)$ such that $\Omega_{h_{C_{i}}}>0$ on each 
irreducible component $C_{i}$. The map $i_{\mathcal{O}_{C}(m C)}: C \rightarrow \mathbb{P}^{N}$ defined by a basis of $H^{0}\left(C, \mathcal{O}_{C}(m C)\right)$ embeds each irreducible component $C_{i}$ if $m \gg 0$, since $C_{i}^{2}>0$ $\left(\mathcal{O}_{C}(m C)\right.$ is the restriction to $C$ of the line bundle associated to the divisor $m C$ on $X)$. If $\tilde{h}$ is a metric on $\mathcal{O}_{\mathbb{P}^{N}}(1)$ with positive $(1,1)$-form $\Omega_{\tilde{h}}$ then the m-square root of the pullback $h^{\prime}=i_{\mathcal{O}_{C}(m C)}^{*} \tilde{h}$ is a metric for $\mathcal{O}_{C}(C)$ satisfying $\Omega_{\left.h^{\prime}\right|_{C_{i}}}>0$ on each $C_{i}$. Let $h$ be a metric of $\mathcal{O}(C)$ on $X$ that is a $C^{\infty}$ extension of $h^{\prime}$. The following steps provide us with a method to modify the metric $h$ and obtain a metric $h^{\prime \prime}$ with the desired curvature properties on a neighborhood of $C$.

Let $F=\left\{x_{k}\right\}_{k=1, \ldots, l}$ be the collection of the normal crossing points of $C$. Choose an open covering $\left\{U_{i}\right\}$ of $X$ consisting of relatively compact open subsets of $X$ with local coordinates $z_{1}^{i}, z_{2}^{i}$ such that the defining equations $f_{i}$ are of the form: 1) $f_{i}=1$, if $\left.C \cap U_{i}=\emptyset ; 2\right) f_{i}=z_{1}^{i}$, if $C \cap U_{i} \neq \emptyset$ and no $x_{k} \in U_{i}$;3) $f_{i}=z_{1}^{i} z_{2}^{i}$, if $C \cap U_{i} \neq \emptyset$ and some $x_{k} \in U_{i}$.

We change $h$ to a new metric $h^{\prime}$ such that $\left.\Omega_{h^{\prime}}\right|_{T_{x_{k}} X}$ is a positive (1,1)-form at the crossing points $x_{k}$. If $x_{k} \in U_{i}$ and the ball $B_{x_{k}}(r)$ is such that $B_{x_{k}}(r) \cap U_{j}=\emptyset$ for $j \neq i$. We change $h_{i}$ to:

$$
h_{i}^{\prime}=h_{i} e^{2 \operatorname{Re}\left(a z_{1} \bar{z}_{2}\right) \cdot \rho}
$$

where $a=-\frac{\partial^{2} \log h_{i}\left(x_{k}\right)}{\partial \bar{z}_{2} \partial z_{1}}$ and $\rho$ is a smooth function on $U_{i}$ with value 1 on $B_{x_{k}}\left(\frac{r}{2}\right)$ and value 0 outside $B_{x_{k}}(r)$. The functions $h_{i}^{\prime}$ and $h_{j}, j \neq i$ give a metric, $h^{\prime}$, on $O(C)$, since $\left.h_{i}^{\prime}\right|_{U_{i} \cap U_{j}}=\left.h_{i}\right|_{U_{i} \cap U_{j}}$ for all $j \neq i$. The new metric $h^{\prime}$ is equal to $h$ on $C,\left.h_{i}^{\prime}\right|_{C \cap U_{i}}=\left.h_{i}\right|_{C \cap U_{i}}$, and has the associated $(1,1)$-form:

$$
\Omega_{h^{\prime}}\left(x_{k}\right)=-\sqrt{-1} \partial_{1} \bar{\partial}_{1} \log h_{i}\left(x_{k}\right)-\sqrt{-1} \partial_{2} \bar{\partial}_{2} \log h_{i}\left(x_{k}\right),
$$

since the modification eliminated the crossed terms. The positivity of $\Omega_{\left.h\right|_{C_{i}}}$ on each $C_{i}$ implies that $-\frac{\partial^{2} \log h_{i}\left(x_{k}\right)}{\partial z_{1} \partial \bar{z}_{1}},-\frac{\partial^{2} \log h_{i}\left(x_{k}\right)}{\partial z_{2} \partial \bar{z}_{2}}>0$ and therefore $\left.\Omega_{h^{\prime}}\right|_{T_{x_{k}} X}$ is a positive (1,1)-form.

To finish, we change $h^{\prime}$ to a metric $h^{\prime \prime}$ which has the curvature form $\Omega_{h^{\prime \prime}}$ positive near the curve $C$. The modification was already done for the smooth case in $[\mathrm{Sc} 73]$ and consists of changing the $h_{i}^{\prime}$ to:

$$
h_{i}^{\prime \prime}=\frac{h_{i}^{\prime}}{1+c h_{i}^{\prime}\left|f_{i}\right|^{2}} .
$$

This change is well defined since the collection functions $1+c h_{i}^{\prime}\left|f_{i}\right|^{2}$ on the $U_{i}$ define a global positive smooth function on $X$. The associated $(1,1)$-form $\Omega_{h^{\prime \prime}}$ changes accordingly to:

$$
\Omega_{h_{i}^{\prime \prime}}=\Omega_{h_{i}^{\prime}}-\sqrt{-1} \partial \bar{\partial} \log \left(1+c h_{i}^{\prime}\left|f_{i}\right|^{2}\right) .
$$

The contribution of $\partial \bar{\partial} \log \left(1+c h_{i}^{\prime}\left|f_{i}\right|^{2}\right)$ on $C \cap U_{i}$ is: 1) $c h_{i}^{\prime} d z_{1}^{i} \wedge d \bar{z}_{1}^{i}$ if $f_{i}=z_{1}^{i}$; 2) $c h_{i}^{\prime}\left|z_{2}^{i}\right|^{2} d z_{1}^{i} \wedge d \bar{z}_{1}^{i}+c h_{i}^{\prime}\left|z_{1}^{i}\right|^{2} d z_{2}^{i} \wedge d \bar{z}_{2}^{i}$ if $f_{i}=z_{1}^{i} z_{2}^{i}$. 
We claim that for $c \gg 0$ this new metric has the desired properties. It is enough to check that for $c \gg 0$ the form $\left.\Omega_{h^{\prime \prime}}\right|_{T_{x} X}$ is positive, i.e. the matrix $\left[\omega_{j k}\right](x)=\left[-\frac{\partial^{2} \log h_{i}^{\prime \prime}}{\partial z_{j}^{i} \partial \bar{z}_{k}^{i}}(x)\right]$ is positive definite, for all $x \in C$.

In case 1) $\left(f_{i}=z_{1}^{i}\right)$ for $x \in C \cap U_{i}$ the off diagonal entries of $\left[\omega_{j k}\right](x)$ and the second diagonal entry do not depend on $c$. On the other hand, the first diagonal entry can be made as big as desired by increasing $c$ making the matrix $\left[\omega_{j k}\right](x)$ positive definite, since the second diagonal entry is positive $\left(\Omega_{\left.h\right|_{C_{i}}}\right.$ is positive). In case 2) $\left(f_{i}=z_{1}^{i} z_{2}^{i}\right)$ the same argument works for the points that are not the crossing point . The positivity of the matrix $\left[\omega_{j k}\right]$ for all $x \in C$ and $c \gg 0$ follows from the compactness of $C$ and the previously established positivity of $\left.\Omega_{h^{\prime}}\right|_{T_{x_{k}} X}$ at the crossing points $x_{k}$.

The following lemma gives two related criteria for the nonembeddability of pseudoconcave ends.

\section{Lemma 2.}

1. Let $X$ be a complex cobordism 2-manifold with $\partial X=M_{1} \amalg M_{0}, M_{1}$ the pseudoconvex end and $M_{0}$ the pseudoconcave end. If $X$ contains a compact positive curve $C \subset X$, then $M_{0}$ is not embeddable.

2. Let $W$ be a pseudoconcave surface containing two disjoint positive curves $C$ and $C^{\prime}$. Then the compact strictly pseudoconvex CR-3-manifold $M=\partial W$ is not embeddable.

Proof. i) If $M_{0}$ is embeddable, then $M_{0}$ is fillable, i.e. $M_{0}=\partial S$ where $S$ is a normal Stein surface [HaLa75]. Let $X^{\prime}$ be the complex space obtained by gluing $S$ to $X$ along $M_{0}, X^{\prime}=X \amalg_{M_{0}} S$. The interior of $X^{\prime}$, $\operatorname{Int}\left(X^{\prime}\right)$, is a strongly pseudoconvex domain and therefore by [Gr62] $\operatorname{Int}\left(X^{\prime}\right)$ is holomorphic convex. That is, $\operatorname{Int}\left(X^{\prime}\right)$ has a proper map to a Stein space $S^{\prime}, r: \operatorname{Int}\left(X^{\prime}\right) \rightarrow S^{\prime}$. Since $S^{\prime}$ can not be a point by the properness of the map $r$, it follows that $\operatorname{Int}\left(X^{\prime}\right)$ has nonconstant holomorphic functions. On the other hand, $\operatorname{Int}\left(X^{\prime}\right)$ contains a pseudoconcave neighborhood $V$ of the positive curve $C$ (i.e $V$ is a compact neighborhood $C$ which is a pseudoconcave manifold) and therefore all holomorphic functions on the $\operatorname{Int}(X)$ must be constant. This follows from the maximum principle and the fact that the pseudoconcavity of $V$ implies that the set $\hat{V}=$ $\left\{x \in \operatorname{Int}\left(X^{\prime}\right)|| f(x)\left|\leq \sup _{y \in V}\right| f(y) \mid\right.$ for all holomorphic functions on $\left.\operatorname{Int}\left(X^{\prime}\right)\right\}$ satisfies $V \subset \operatorname{Int}(\hat{V})[$ AnGr62].

ii) Let $V$ be a pseudoconcave neighborhood of $C$, then $X=W \backslash \operatorname{Int}(V)$ is a complex cobordism manifold with $M=\partial W$ as its pseudoconcancave end. Since $X$ contains the positive curve $C^{\prime}$, by i) $M$ is not embeddable.

The previous lemmas plus the construction of pseudoconcave surfaces containing disjoint positive curves will give the proof of the main theorem:

Theorem 1. There are complex cobordant 3-dimensional strictly pseudoconvex CR-manifolds $M_{1}$ and $M_{0}$, with one embeddable and the other not. Equivalently, the embeddability of CR-3-manifolds is not a complex cobordism invariant. 
Proof. First, we describe a process to construct a pseudoconcave surface $W$ containing two positive curves $C$ and $C^{\prime}$ that do not intersect.

Let $C_{1}, C_{2}$ and $C_{3}$ be 3 positive curves in the smooth projective surfaces $X_{1}, X_{2}$ and $X_{3}$ respectively. First, we glue a neighborhood of $C_{1}$ in $X_{1}$ with a neighborhood of $C_{2}$ in $X_{2}$ obtaining a surface $Y$ containing $C_{1}$ and $C_{2}$, with equivalent neighborhood germs, intersecting transversely at one point.

To do this pick a point $p_{1} \in C_{1}$ and let $U_{1}$ be an open subset of $X_{1}$ containing $p_{1}$ and with a biholomorphism $\phi: U_{1} \rightarrow \Delta \times \Delta, \Delta$ the disc of radius 1 , such that $\phi\left(C_{1} \cap U_{1}\right)=0 \times \Delta$. Also, let $U_{2}$ be an open subset of $X_{1}$ such that $C_{1} \subset U_{1} \cup U_{2}$ and $\phi\left(U_{1} \cap U_{2}\right) \subset \Delta_{\frac{1}{2}} \times\left(\Delta \backslash \Delta_{\frac{1}{2}}\right), \Delta_{\frac{1}{2}}$ the disc of radius $1 / 2$. Do the same for $C_{2}$ in $X_{2}$. Pick a point $p_{2} \in C_{2}$, an open subset $V_{1} \subset X_{2}$ and a biholomorphism $\varphi: V_{1} \rightarrow \Delta \times \Delta$ such that $\varphi\left(C_{2} \cap V_{1}\right)=\Delta \times 0$. Pick $V_{2}$ such that $C_{2} \subset V_{1} \cup V_{2}$ and $\varphi\left(V_{1} \cap V_{2}\right) \subset\left(\Delta \backslash \Delta_{\frac{1}{2}}\right) \times \Delta_{\frac{1}{2}}$. The surface $Y$ is the surface given by the open sets $\Delta \times \Delta, U_{2}$ and $V_{2}$ with the gluings of $U_{2}$ and $V_{2}$ with $\Delta \times \Delta$ given by respectively $\left.\phi\right|_{U_{1} \cap U_{2}}$ and $\left.\varphi\right|_{V_{1} \cap V_{2}}$.

Repeat the same gluing argument for neighborhoods of the curve $C_{1} \cup C_{2}$ in $Y$ and the curve $C_{3}$ in $X_{3}$ (picking a different crossing point). The resulting surface $Y^{\prime}$ contains the normal crossing divisor $\tilde{C}=C_{1} \cup C_{2} \cup C_{3}$ and the neighborhood germs of $C_{1}, C_{2}$ and $C_{3}$ in $Y^{\prime}$ are equivalent to the neighborhood germs of $C_{1}$, $C_{2}$ and $C_{3}$ in $X_{1}, X_{2}$ and $X_{3}$ respectively. The surface $Y^{\prime}$ and the curves $C_{1}$, $C_{2}$ and $C_{3}$ satisfy the conditions of Lemma 1 , hence there is a pseudoconcave neighborhood $V$ of $\tilde{C}=C_{1} \cup C_{2} \cup C_{3}$ on $Y^{\prime}$. The desired pseudoconcave surface $W$ and disjoint positive curves $C$ and $C^{\prime}$ will be respectively $V, C_{1}$ and $C_{3}$.

Consider the cobordism manifold $X=W \backslash \operatorname{Int}\left(V_{1}\right)$, where $V_{1}$ is a pseudoconcave neighborhood of $C$ in $W \cap\left(U_{1} \cup U_{2}\right)$. The pseudoconcave end $M_{0}=\partial W$ is nonembeddable by Lemma 2 . The pseudoconvex end $M_{1}=\partial V_{1}$ is embeddable since it is fillable, $V_{1}$ is also a pseudoconcave neighborhood of $C_{1}$ in the compact surface $X_{1}$.

A pseudoconcave manifold $Y$ is embeddable if there is an embedding of $Y$ in a compact complex manifold without boundary $X$. The problem of preservation of embeddability by complex cobordisms is equivalent to the question of whether a pseudoconcave extension $Y$ of an embeddable pseudoconcave manifold $Y^{\prime}$ is also embeddable. $X$ is embeddable if and only if $\partial Y$ is fillable, or equivalentely the complex cobordism $Y \backslash \operatorname{Int}\left(Y^{\prime}\right)$ preserves embeddalility of its ends. Hence it follows from Theorem 1:

Corollary 1. There is a nonembeddable pseudoconcave surface $Y$ containing an embeddable pseudoconcave surface $Y^{\prime}$.

In the proof of the theorem, we constructed a pseudoconcave surface $W$ with two disjoint positive curves (repeating the construction ad libitum we could have produced as many disjoint positive curves as desired). If we take out from $W$ a sufficiently small pseudoconcave neighborhood of each of the disjoint positive curves, we obtain: 
Corollary 2. There are complex cobordism manifolds $X$ of dimension 2 with more than one pseudoconvex end (impossible in higher dimensions).

Next we consider the problem of whether a CR-function on the pseudoconvex end of a complex manifold with boundary $X$ is the boundary value of a holomorphic function on $X$. We are interested in the case where all the boundary components of $X$ are strictly pseudoconvex CR-manifolds. The relevance of this boundary value problem comes from the fact that if $X$ has dimension greater than 2 then all CR-functions on the pseudoconvex end are boundary values of a holomorphic function on $X$. The embeddability of all (2n-1)-dimensional strictly pseudoconvex CR-manifolds for $n>2$ implies that all pseudoconcave ends of $X$ are fillable. Hence $X$ is an open subset of a filling (pseudoconvex) of its pseudoconvex end. In [KoRo65] it is shown that all CR-functions on the smooth boundary of a strictly pseudoconvex domain are extendable to holomorphic functions on the domain.

Proposition 1. The extendability of CR-functions on the pseudoconvex end of a complex cobordism manifold $X$, whose ends are all strictly pseudoconvex $C R$ manifolds, to holomorphic functions on $X$ does not need to hold if dimension of $X$ is 2 .

Proof. The cobordism manifold $X$ described in the proof of the theorem implies the Proposition. Its pseudoconvex end $M_{1}$ is embeddable and therefore it has many nonconstant CR-functions and since $X$ contains a positive curve inside $\left(C_{3}\right.$ in the example) all global holomorphic functions on $X$ must be constant as in Lemma 2 i).

\section{How "good" can a "bad" complex cobordism be?}

The examples of complex cobordism manifolds described in the proof of Theorem 1 are very different from the complex cobordism manifolds that preserve embeddability. The results in this section go in the opposite direction. A complex cobordism manifold $X$ preserving embeddability must satisfy the condition that all CR-functions on the pseudoconvex end $M_{1}$ extend to holomorphic functions on $X$. As mentioned earlier, this condition also played an essential role in the proof of the preservation of embeddability for strict CR-cobordisms [EpHe01]. Our examples of complex cobordisms above, where embeddability is not preserved, strongly violated the extendability of CR-functions. Theorem 2 shows that the extendability of CR-functions on the pseudoconvex end is necessary but not sufficient for a complex cobordism to preserve embeddability. Later in this section, in Theorem 3, it is shown that there are small deformations of a complex cobordism 2-manifold preserving embeddability that no longer preserve embeddability.

Theorem 2. There are complex cobordism 2-manifolds $X$ whose pseudoconvex end $M_{1}$ is embeddable and all CR-functions on $M_{1}$ extend to holomorphic functions on $\operatorname{Int}(X)$, yet the pseudoconcave end $M_{0}$ is not embeddable. 
Proof. Consider three linear $\mathbb{P}^{1} \subset \mathbb{P}^{2} C_{1}, C_{2}$ and $C_{3}$ such that $C=C_{1} \cup C_{2} \cup C_{3}$ is a normal crossing divisor. Let $U_{1}, U_{2}$ and $U_{3}$ be tubular neighborhoods for respectively $C_{1}, C_{2}$ and $C_{3}$ such that $U_{1} \cap U_{2} \cap U_{3}=\emptyset$. Let $W^{\prime}$ be the complex surface that results from $U_{1} \cup U_{2} \cup U_{3} \subset \mathbb{P}^{2}$ by separating $U_{2}$ from $U_{3}$. $W^{\prime}$ has a natural holomorphic map to $\mathbb{P}^{2}, p: W^{\prime} \rightarrow \mathbb{P}^{2}$ which identifies the points that were separated.

The surface $W^{\prime}$ and curves in $C_{1}^{\prime}, C_{2}^{\prime}$ and $C_{3}^{\prime}$ in $W^{\prime}$ coming from the three linear $\mathbb{P}^{1}, C_{1}, C_{2}$ and $C_{3}$, in $\mathbb{P}^{2}$ satisfy the conditions stated in Lemma 1 and $C_{2}^{\prime} \cap C_{3}^{\prime}=\emptyset$. Lemma 1 implies that there is a pseudoconcave surface $W \subset W^{\prime}$ containing the curve $C_{1}^{\prime} \cup C_{2}^{\prime} \cup C_{3}^{\prime}$. Since $W$ contains 2 disjoint positive curves $C_{2}$ and $C_{3}, \partial W$ can not be embeddable by Lemma 2 ii).

After making the identification of $\mathbb{P}^{2} \backslash C_{1}$ with $\mathbb{C}^{2}$, consider a sufficiently large ball $B \in \mathbb{C}^{2}$ such that $\mathbb{P}^{2} \backslash B$ is contained in $U_{1}$. It follows that $\mathbb{P}^{2} \backslash B$ can also be considered as a subset of $W^{\prime}$. If one considers an even larger ball $B^{\prime}$, one can have $\left(\mathbb{P}^{2} \backslash B^{\prime}\right) \subset W$. The pseudoconcave surface $V=\mathbb{P}^{2} \backslash B^{\prime}$ is naturally embedded as neighborhood of $C_{1}$ in $\mathbb{P}^{2}$ or as a neighborhood of $C_{1}^{\prime}$ in $W$. The open subset $X=W \backslash \operatorname{Int}(V)$ is a complex cobordism with an embeddable pseudoconvex end $M_{1}=\partial V$, isomorphic to the boundary of the ball $B^{\prime} \subset \mathbb{C}^{2}$, and a nonembeddable pseudoconcave end $M_{0}=\partial W$.

We conclude by showing that all CR-functions on $M_{1}$ extend to holomorphic functions of $\operatorname{Int}(X)$. The map $p: W^{\prime} \rightarrow \mathbb{P}^{2}$ gives a CR-isomorphism of $M_{1}$ with $\partial B^{\prime}$ and a holomorphic map $\left.p\right|_{X}: X \rightarrow B^{\prime}$. Any CR-function, $\varphi$, on $M_{1}$ is the pullback by $p$ of a CR-function, $\varphi^{\prime}$, on $\partial B^{\prime}$. The holomophic function on $X$ extending the CR-function, $\varphi$, on $M_{1}$ will be the pullback by $p$ of the holomorphic function on the ball $B^{\prime}$ extending $\varphi^{\prime}$.

In order to express a consequence of Theorem 2, we introduce two weakened versions of embeddability of a pseudoconcave surface $X_{-}$described in [EpHe2-00].

Let $X_{-}$be a pseudoconcave surface with a positive curve $Z$. The pair $\left(X_{-}, Z\right)$ is called weakly embeddable if the holomorphic map defined by the sections of $H^{0}\left(X_{-}, \mathcal{O}(d Z)\right), \varphi_{d}: X_{-} \rightarrow \mathbb{P}^{n}$, is injective in some neighborhood of $Z$ for sufficiently large $d$. The pair $\left(X_{-}, Z\right)$ is called almost embeddable if in addition the map $\varphi_{d}: X_{-} \rightarrow \mathbb{P}^{n}$ is an embedding outside a proper analytic subset of $X_{-} \backslash Z$. In [EpHe2-00] the authors ask about the relationship between these two notions of weakened embeddability. The interest sprouted from the fact that one has an operative numerical criterion involving the dimensions $h^{0}\left(X_{-}, \mathcal{O}(d Z)\right)$ characterizing weak embeddability and that almost embeddability of $X_{-}$implies embeddability of $\partial X_{-}$. In the proof of Theorem 2 we constructed the weakly embeddable pair $\left(W, C_{1}\right)$ such that for any $d$ the maps $\varphi_{d}: W \rightarrow \mathbb{P}^{n}$ can not distinguish any pair points in $W$ that came from the same point in $U_{2} \cap U_{3}$ and we have:

Corollary 3. The weak embeddability of a pseudoconcave surface $\left(X_{-}, Z\right)$ does not imply that $\left(X_{-}, Z\right)$ is almost embeddable. 
The following result states that for a complex cobordism manifold the property of preserving embeddability is not stable under small deformations. We need to recall some facts about the moduli spaces holomorphic neighborhood retracts (HNR) of a curve on a surface for our proof of Theorem 3.

An embedding of a curve $C$ in a manifold $X$ is a HNR if there is a neighborhood $W$ of $C$ with a holomorphic map $r: W \rightarrow C$ such that $\left.r\right|_{C}=\operatorname{Id}_{C}$. The space of nonequivalent HNR embeddings of a curve $C$ with normal bundle a given line bundle $L$ is described in [MoRo81]. Let $\{U, V\}$ be a covering of $C$ such that $U$ is a disc and $V \cap U$ is an annulus. A HNR embedding of $C$ with normal bundle $L$ can be constructed by glueing $U \times \mathbb{C}$ with $V \times \mathbb{C}$ via the map $\phi=(\mathrm{Id}, f): U \cap V \times \mathbb{C} \rightarrow U \cap V \times \mathbb{C}$,

$$
f(z, u)=g(z)\left(u+\sum_{i \geq 1} f_{i}(z) u^{i+1}\right)
$$

where $z$ is the coordinate of $U \cap V \subset U$ and $g, f_{i} \in \mathcal{O}(U \cap V), g$ is the transition function for the normal bundle $L$. To the HNR described above we can associate a point in $E\left(\bigoplus_{i>0} H^{1}\left(C, L^{-i}\right)\right)$, the weighted projective space with the $\mathbb{C}^{*}$ action $s .\left(x_{1}, x_{2}, \ldots, x_{n}, \ldots\right)=\left(s x_{1}, s^{2} x_{2}, \ldots, s^{n} x_{n}, \ldots\right)$. This is done by considering the $f_{i}$ as cocycles representing classes of $H^{1}\left(C, L^{-i}\right) \simeq \mathcal{O}(U \cap V) / \delta(\mathcal{O}(V) \oplus \mathcal{O}(U))$. [MoRo81] shows that the neighborhood germs of two HNRs of $C$ with normal bundle $L$ are equivalent only if the corresponding points in $E\left(\bigoplus_{i>0} H^{1}\left(C, L^{-i}\right)\right)$ coincide.

Theorem 3. There are holomorphic families of complex cobordism 2-manifolds where all the members have their pseudoconvex ends embeddable, but only the central member has the pseudoconcave end embeddable.

Proof. We will construct a family of open surfaces such that:

1. each member contains two $\mathbb{P}^{1}$ 's,$C_{1}$ and $C_{2}$, with normal bundle $\mathcal{O}(1)$ intersecting at one point;

2. only one member has the neighborhood germ of $C_{1}$ union with $C_{2}$ equivalent to the neighborhood germ of the union of two linear $\mathbb{P}^{1}$ 's in $\mathbb{P}^{2}$.

Let $C_{1}$ and $C_{2}$ be two distinct linear $\mathbb{P}^{1}$ 's in $\mathbb{P}^{2}$ and $\left\{x_{0}\right\}=C_{1} \cap C_{2}$. Let $U_{0}, U_{1}$ and $U_{2}$ be a covering of a neighborhood $U$ of $C=C_{1} \cup C_{2}$ such that $x_{0} \in U_{0}, C_{1} \subset U_{0} \cup U_{1}, C_{2} \subset U_{0} \cup U_{2}$, and $U_{1} \cap U_{2}=\emptyset$. Since the neighborhood germs of a linear $\mathbb{P}^{1}$ in $\mathbb{P}^{2}$ and of the zero section of the line bundle $\mathcal{O}(1)$ over $\mathbb{P}^{1}$ are equivalent, we can choose $U_{0}$ and $U_{2}$ to be biholomorphic to the bidisc, $U_{0} \cap C_{2}=\Delta \times 0, U_{2} \cap C_{2}=\Delta \times 0$ and $U_{0} \cap U_{2} \subset\left(\Delta \backslash \Delta_{\frac{1}{2}}\right) \times \Delta \subset U_{0}$. Moreover, we can describe $U_{0} \cup U_{2}$ as the complex surface resulting from glueing $U_{0}$ and $U_{2}$ via the map $\phi=(\operatorname{Id}, f)(z, u):\left(U_{0} \cap U_{2} \cap C_{2}\right) \times \Delta \rightarrow\left(U_{0} \cap U_{2} \cap C_{2}\right) \times \Delta$, where $f(z, u)=g(z) u$ and $g$ is the transition function for the line bundle $\mathcal{O}(1)$.

Change the glueing map of $U_{0}$ and $U_{2}$ by varying $f(z, u)$ holomorphically with respect to a parameter $\mathrm{t}, f_{t}(z, u)$, by introducing nontrivial higher order terms $f_{i, t}, i>1$. From the description in the paragraph before the theorem, it follows 
that by making $f_{i, t} \in H^{1}\left(\mathbb{P}^{1}, \mathcal{O}(1)\right) \neq 0$ for $t \neq 0$ the resulting holomorphic family of HNR of $\mathbb{P}^{1}$ is such that only for $t=0$ the HNR is equivalent to the linear $\mathbb{P}^{1}$ in $\mathbb{P}^{2}$.

Let $\omega: \mathcal{V} \rightarrow \Delta$ be the family of surfaces, with $\mathcal{V}_{t}=V_{t}$ and $V_{0}=U$, obtained by keeping the gluing of $U_{0}$ with $U_{1}$ but changing the gluing of $U_{0}$ with $U_{2}$ as in the previous paragraph. Each member $V_{t}$ of the family has the curve $C=C_{1} \cup C_{2}$ embedded with the same normal bundle. Hence the germ of the tubular neighborhood of $C$ in all the $V_{t}$ is diffeomorphic to the germ of the tubular neighborhood $T$ of $C$ in $V_{0}$. It follows that there is a smooth map $\phi: T \times \Delta \rightarrow \mathcal{V}$, with each $\phi_{t}: T \rightarrow T_{t}=\phi(T \times t) \subset V_{t}$ a diffeomorphism from $T$ to a tubular neighborhood of $C$ in $V_{t}$ sending $C$ in $T$ to $C$ in $V_{t}$. The union of all $T_{t}, \mathcal{T}=\bigcup_{t \in \Delta} T_{t} \subset \mathcal{V}$, gives a family of surfaces $\left.\omega\right|_{\mathcal{T}}: \mathcal{T} \rightarrow \Delta$ (the family $\mathcal{T}$ can also be perceived as the variation of the complex structure induced by the diffeomorphisms $\phi_{t}$ on differential manifold $T$ ).

By Lemma 1 there is a strictly plurisubharmonic function $g: V_{0} \backslash C \rightarrow \mathbb{R}$ such that $\left\{x \in V_{0}: g(x) \geq c\right\} \subset T$ for $c \gg 0$ and $S_{c}=\{x \in T: g(x)=c\}$ is a compact SPCR-3-manifold. After possibly shrinking $\Delta, c \gg 0$ can be such that $g_{t}=g \circ \phi_{t}^{-1}: T_{t} \backslash C \rightarrow \mathbb{R}$ is a strictly plurisubharmonic on a neighborhood of $\phi_{t}\left(S_{c}\right)$ for all $t \in \Delta$. Let $Y_{t}=\left\{x \in T_{t} \mid g_{t}(x)>c\right.$ or $\left.x \in C\right\} \subset T_{t}$, the union of all $Y_{t}, \mathcal{Y}=\bigcup_{t \in \Delta} Y_{t} \subset \mathcal{V}$, gives a family, $\omega \mid \mathcal{Y}: \mathcal{Y} \rightarrow \Delta$, of pseudoconcave neighborhoods of $C=C_{1} \cup C_{2}$.

We claim that the boundaries, $\partial Y_{t}$, of the pseudoconcave surfaces $Y_{t}$ are not embeddable for $t \neq 0$. By construction, $Y_{t}$ contains the rational curve $C_{2}$ with normal bundle of degree one but whose embedding is not equivalent to the embedding of the linear $\mathbb{P}^{1} \subset \mathbb{P}^{2}$ for $t \neq 0$. If a $\partial Y_{t}$ were embeddable for $t \neq 0$ then $Y_{t}$ would be contained in a smooth algebraic surface $S_{t}$ coming from filling $\partial Y_{t}$ and resolving the singularities. The claim follows from the result 6.13 of [MoRo81], which proves that the only neighborhood germ of $\mathbb{P}^{1}$ with normal bundle $\mathcal{O}(1)$ that can be embedded in an algebraic surface is the neighborhood germ of the linear $\mathbb{P}^{1} \subset \mathbb{P}^{2}$.

To construct the family of complex cobordisms, we notice that all $Y_{t}$ contain a pseudoconcave neighborhood of $C_{1}$ whose boundary is embeddable. By Lemma 1 , we can choose a sufficiently small pseudoconcave neighborhood $W \subset V_{0}$ of $C_{1}$ such that $W_{t}=\phi_{t}(W)$ gives a family of pseudoconcave surfaces, $\left.\omega\right|_{\mathcal{W}}$ : $\mathcal{W} \rightarrow \Delta$. All members of the family $\mathcal{W}$ are biholomorphic to the pseudoconcave neighborhood $W \subset \mathbb{P}^{2}$ of $C_{1}$, therefore their pseudoconcave ends $\partial W_{t} \simeq \partial W$ are embeddable.

The family of complex cobordism manifolds $\left.\omega\right|_{\mathcal{X}}: \mathcal{X}=\mathcal{Y} \backslash \operatorname{Int}(\mathcal{W}) \rightarrow \Delta$ gives the desired example. The pseudoconvex end $M_{0_{t}}=\partial W_{t}$ of $X_{t}$ is CR-isomorphic to the embeddable SPCR-3-manifold $\partial W$ but the pseudoconcave end $M_{1_{t}}=\partial Y_{t}$ is not embebeddable for $t \neq 0$, as was explained above. 
Remark. The example in the proof also shows that: the extendability of the CR-functions of the pseudoconvex end to holomorphic functions on the complexcobordism manifold is a property that is not locally stable in families of complexcobordisms. Suppose all CR-functions on $\partial W_{t}$ extend to holomorphic functions on $X_{t}$, then the natural fixed inclusion $W_{t} \subset \mathbb{P}^{2}$ would extend to holomorphic maps $i_{t}: Y_{t} \rightarrow \mathbb{P}^{2}$. For $t \neq 0$ sufficiently small, $i_{t}$ is a small perturbation of $i_{0}$. Therefore CR-map of $\partial Y_{t}$ to $\mathbb{C}^{2}$ induced by $i_{t}$ is an embedding since it is a small peturbation of CR-embedding of $\partial Y_{0}$ in $\mathbb{C}^{2}$, which gives the desired contradiction.

It is well known that the embeddability of strictly pseudoconvex CR-structures on a 3-manifold is not stable under small deformations of the CR-structure. It follows from the proof of the theorem that the instability of the embeddability property still holds under the condition that all deformed CR-structures are complex cobordant to a fixed embeddable SPCR-3-manifold.

Corollary 4. Let $M_{1}$ be an embeddable SPCR-3-manifold. The embeddability of SPCR-3-manifolds complex-cobordant to $M_{1}$ is not stable, for small deformations of the CR-structure preserving the property of being complex-cobordant to $M_{1}$.

Proof. In the proof of theorem 3 all the SPCR-3-manifolds $M_{1 t}$ are complex cobordant to $S^{3}$ with the canonical embeddable CR-structure.

\section{Acknowledments}

I would like to thank Professor Charles Epstein for generously sharing his knowledge with me and for his enthusiasm and Professor Yum-Tong Siu for always inspiring and encouraging me and the hospitality of the staff at Harvard University. I would also like to thank the referee for his comments

\section{References}

[AnGr62] Andreotti, A., Grauert, H. Théorèmes de finitude pour la cohomologie des espaces complexes. Bull. Soc. Math. France, 90,(1962),193-259.

[B194] Bland, J. Contact geometry and CR structures on $S^{3}$. Acta Math., 172,(1994),1-49.

[Bou74] Boutet de Monvel, L. Intégration des équations de Cauchy-Riemann induites. Seminaire Goulaoic-Lions-Schwartz, Exposé IX, (1974-1975)

[BuEp90] Burns, D., Epstein, E. Embeddability for three-dimensional CR-manifolds. J. Amer. Math. Soc, 3, (1990), 809-841.

[EpHe1-00] Epstein, C., Henkin, G. Embeddings for 3-dimensional CR-manifolds. Progress in Math., 188, Birkhauser (2000).

[EpHe2-00] , Stability of embeddings for pseudoconcave surfaces and their boundaries. Acta Math., 185, (2000), 161-237.

[EpHe01] _ Can a good manifold come to a bad end?. Proc. Steklov Inst., 235, (2001)

[Gr62] Grauert, H. Über Modifikationen und exzeptionelle analytische Mengen. Math. Ann, 146,(1962),331-368.

[HaLa75] Harvey, R., Lawson, H. On the boundaries of complex analytic varieties I. Ann. Math. (2), 102, (1975), 223-295. 
[Ko86] Kohn, J. The range of the tangential Cauchy-Riemann operator. Duke Math. J., 53, (1986), 525-545.

[KoRo65] Kohn, J., Rossi, H. On the extension of holomorphic functions from the boundary of a complex manifold. Ann. Math. (2), 81, (1965), 451-472.

[Le94] Lempert, L. Embeddings of three dimensional Cauchy-Riemann manifolds. Math. Ann., 300, (1994), 1-15.

[Le95] _ _ Algebraic approximations in analytic geometry. Inv. Math, 121, (1995), 335353.

[Ro65] Rossi, H. Attaching analytic spaces to an analytic space along a pseudoconcave boundary., Proc. Conf. on Complex Manifolds (Minneapolis, 1964), Springer, Berlin, 1965, $242-256$.

[MoRo81] Morrow, J., Rossi, H. Some general results on equivalence of embeddings. Princeton Annals, Proceedings of 1979 Princeton Conference on Complex Analysis, 1981, 299-325.

[Sc73] Schneider, M. Über eine Vermutung von Hartshorne. Math. Ann, 201,(1973),221-229.

University of Miami, Department of Mathematics, Coral Gables, FL 33124

E-mail address: bdeolive@math.miami.edu 